\title{
Potencial visual evocado por padrão reverso com tabuleiro de xadrez em gatos domésticos adultos
}

\author{
Pattern reversal visual evoked potential using checkerboard stimulus in adult domestic cats
}

\author{
Nathalia Veronica Carneiro Ferreira ${ }^{\mathrm{I}}$ Elton Hugo Lima da Silva Souza ${ }^{\mathrm{I}}$ \\ Jéssica Karla Alves da Silva ${ }^{I}$ Valdir Luna da Silva ${ }^{\text {II }}$ \\ Bruno Oliveira Ferreira de Souza ${ }^{\mathrm{III}}$ Fabrício Bezerra de Sá ${ }^{*}$
}

\section{RESUMO}

O potencial visual evocado por padrão reverso (PVE-PR) é um método objetivo, não invasivo e estabelecido para as investigações funcionais. Este trabalho teve como objetivo a obtenção de dados do PVE-PR com tabuleiro de xadrez em gatos domésticos adultos, além de verificar a influência de diferentes angulos visuais sobre os picos de tempo implícito e amplitude das ondas N75 e P100. Foram utilizados 13 gatos adultos (2-4 anos de idade), de ambos os sexos. Cada animal foi posicionado $100 \mathrm{~cm}$ de distância de um monitor de vídeo situado a 17,2 $2^{\circ}$. O estímulo gerado para a formação dos PVEs foi uma onda "quadrada" com a frequência de $6 \mathrm{~Hz}$, apresentados em cinco frequências espaciais (16', 32', 64', 130'e 260'de arco). Os valores médios dos tempos implícitos (ms) e amplitudes $(\mu \mathrm{V})$ de P100 não foram diferentes estatisticamente e nem estavam correlacionados $(r=0,067)$. Com a mudança de tamanho do ângulo de estímulo apresentado, os picos de tempo implícito e amplitude do P100 apresentaram uma discreta variação. Aos 32' de arco, a amplitude de P100 atingiu seu pico

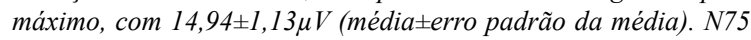
não mostrou nenhuma variação de amplitude. Com 16'de arco,o tempo implícito de N75 diminuiu, não havendo correlação com os valores de amplitude $(r=0,26)$. O ângulo visual que desencadeou um maior pico de amplitude e tempo implícito constante para P100, foi o de 32' de arco. Em gatos adultos, um PVE-PR pode ser obtido sem o uso de midriáticos e sedativos, com estímulos subtendidos entre ângulos grandes como 64' e pequenos como 16'.

Palavras-chave: felinos, teste eletrofisiológico, oftalmologia.

\section{ABSTRACT}

The pattern reversal visual evoked potential (PR$V E P)$ is an objective method, stablished for noninvasive functional investigations. This research aimed to obtain data from the PR$V E P$ with checkerboard in adult domestic cats and the influence of different visual angles on the peak latency and implicit time of waves N75 and P100. It was used 13 adult cats (2-4 years old), of both sexes. Each animal was positioned $100 \mathrm{~cm}$ away from a video monitor located at $17.2^{\circ}$. The stimulus generated for the formation of wave VEPs was "square" with the frequency of $6 \mathrm{~Hz}$, presented in five spatial frequencies (16 ', 32',64', 130' and 260 'of arc). Mean values of implicit times ( $\mathrm{ms}$ ) and amplitudes ( $\mu$ volts) of P100 were not statistically different and were not correlated $(r=0.067)$. With the change in size of the angle of stimulus presented, the peak implicit time and amplitude of P100 showed a slight variation. At 32 'of arc amplitude of $P 100$ reached its peak with 14,94 $\pm 1,13 \mu \mathrm{V}$ (mean $\pm S E M)$. N75 showed no variation in amplitude. With 16'of arc the implicit time N75 decreased and no correlation with amplitude values was observed $(r=0,26)$. The visual angle that triggered higher peak amplitude and implicit time constant for P100, was 32'of arc. In adult cats, PR-VEP can be obtained without mydriatic and sedatives, with stimuli subtending angles between large as $64^{\prime}$ and small as 16 '.

Key words: feline, electrophysiological test, ophthalmology.

\section{INTRODUÇÃO}

Os testes visuais nos animais podem ser realizados de várias maneiras, como métodos comportamentais (MILLER \& MURPHY, 1995; GEARHART et al., 2008), discriminação de formas e aprendizagens (TANAKA, 2000), nistagmo optocinético (EZHE et al., 1990), eletrorretinograma (ERG) (ROSOLEN et al., 2005) e potencial visual evocado (PVE) (MURPHY et al., 1997).

O potencial visual evocado (PVE) é um método objetivo, não invasivo e estabelecido para

\footnotetext{
${ }^{\mathrm{I} U n i v e r s i d a d e ~ F e d e r a l ~ R u r a l ~ d e ~ P e r n a m b u c o ~(U F R P E), ~ 52171-900, ~ R e c i f e, ~ P E, ~ B r a s i l . ~ E-m a i l: ~ c r l e u c a s @ y a h o o . c o m . ~ * A u t o r ~ p a r a ~}$ correspondência.

IIUniversidade Federal de Pernambuco, Recife, Brasil.

IIIEscola de Optometria, Universidade de Montreal, Montreal, Quebec, Canadá.
} 
investigações da função visual (JACQUES et al., 2011). Ele está formado por sinais extraídos da atividade elétrica do córtex visual, após impulsos serem gerados em um campo visual central da retina, seguindo pelo nervo óptico, passando pelo corpo geniculado lateral, com radiação óptica até o córtex occipital (OYAMADA, 2000; SALOMÃO, 2007; ODOM et al., 2010). OPVE de varredura é comumente empregado para identificar disfunções no sistema visual, resultante de doenças desmielinizantes, neurite óptica, neuropatias isquêmicas e compressivas, além de servir como recurso para obtenção da Acuidade Visual (AV) em pacientes não verbais e não cooperativos, como crianças e animais (REN et al., 2000). Além de ser utilisado como ferramenta para o diagnóstico de neuropatias, o potencial visual evocado por padrão reverso (PVE-PR) com tabuleiro de xadrez serve como ferramenta para avaliar o impacto destas doenças sobre a função visual em seres humanos (JUERGEN et al., 2014). Na prática da oftalmologia veterinária, o ERG é aplicado rotineiramente como exame eletrofisiológico de escolha no diagnóstico de diferentes retinopatias. O PVE é frequentemente utilizado para obter valores de AV em animais de companhia (MURPHY et al., 1997; OYAMADA, 2000; MORENO-CARMONA, 2006). Diferentes estudos contribuíram para a normatização de valores de AV para cães adultos e jovens em diferentes idades (MORENO-CARMONA, 2006) e em animais anestesiados (ITOH, 2010). A despeito disso, o uso do PVE como auxílio ao diagnóstico de neuropatias e de afeç̧ões das vias visuais centrais na clínica oftálmica veterinária ainda é escasso.

Em gatos, estudos com PVE foram realizados com capturas de sinais diretamente do córtex visual, por meio de eletrodos implantados cirurgicamente em áreas específicas do lobo occipital (SCHMIDT et al., 2004). Entretanto, o relato de registros eletrofisiológicos de PVEs em condições clínicas nesta espécie são raros.

Considerando a existência de poucos relatos e valendo-se da importância de se obter dados para a padronização de uma técnica de eletrodiagnóstico em gatos domésticos adultos, o presente estudo tem como objetivo registrar dados do PVE por reversão de padrões, sem o uso de anestésicos e midriáticos, utilizando eletrodos de scalp.

\section{MATERIAL E MÉTODOS}

\section{Animais}

Foram usados 13 gatos, adultos jovens ( 2 - 4 anos de idade), 6 machos e 7 fêmeas, sem raça definida, clinicamente sadios, que não apresentavam sinais clínicos de doença oftálmica, atendidos no Hospital de Medicina Veterinária da Universidade Federal Rural de Pernambuco. Previamente à realização do PVE$\mathrm{PR}$, os animais foram examinados seguindo a marcha de exame oftálmico, iniciado pelo teste lacrimal de Schirmer e reflexos pupilares diretos e consensuais, seguido pela oftalmoscopia direta e indireta.

\section{Equipamento e estímulos}

O Sistema utilizado é constituído pelo Nihon Kohdem, Neuropack 2 MEB-7102A/k, com o seu sinal digitalizado pelo DATAQ ${ }^{\circledR}$ DI-158U, por meio de um cabo RS232 com dois canais e acoplado a um notebook Acer Aspire 4732Z-4620. Os potenciais visuais evocados foram obtidos com estímulos de reversão de padrões do tipo tabuleiro de xadrez. O estímulo gerado para a formação dos PVEs foi uma onda "quadrada" com a frequência de $6 \mathrm{~Hz}$, apresentados em cinco frequências espaciais $\left(16^{\prime}, 32^{\prime}, 64^{\prime}, 130^{\prime}\right.$ e $260^{\prime}$ de arco). Foi projetado em um monitor de vídeo, com luminância máxima de $100 \mathrm{~cd} \mathrm{~m}^{-2}$, com taxa de reversão de $1.0 \mathrm{~Hz}$, posicionado a uma distância de $100 \mathrm{~cm}$ de cada animal e subtendido a $17,2^{\circ}$ dos olhos dos animais (Figura 1). A luminância ambiente foi mantida a $30 \mathrm{~cd}$ $\mathrm{m}^{-2} \mathrm{e}$ aparelhos eletrônicos não necessários para a realização dos exames foram desligados para evitar ruídos e artefatos. Para cada frequência espacial, foram realizadas dez repetições com duração de um segundo cada. As respostas foram obtidas a partir de estímulos binoculares e, para captura, foram utilizados eletrodos de contato da Nihon Kohdem, modelo NE-124 conectados ao Electrode Junction Box JB-711B do Neuropack 2 MEB-7102A/k.

Posicionamento de eletrodos e registro eletrofisiológico

Após a higienização com solução desengordurante $(70 \%$ éter P.A. e $30 \%$ álcool absoluto), utilizou -se pasta condutora nos locais a serem posicionados os eletrodos. Os PVE-PRs foram registrados a partir de eletrodos bipolares ativos, $\mathrm{O} 1$ (occipital 1, esquerdo) e $\mathrm{O} 2$ (occipital 2, direito), com $2-3 \mathrm{~cm}$ de distância um do outro, $(\mathrm{Fpz})$ um eletrodo de referência comum, colocado na linha mediana do crânio há $1,2 \mathrm{~cm}$ acima da articulação fronto nasal e um eletrodo terra (A1), posicionado na região auricular (Figura 1). É importante evidenciar que os animais não foram sedados e que não foram utilizados midriáticos. Os registros do PVE-PR estão constituídos por três picos, denominados negativos e positivo, N75, P100 e N135 (ODOM, 2010). Foram

Ciência Rural, v.45, n.5, mai, 2015. 


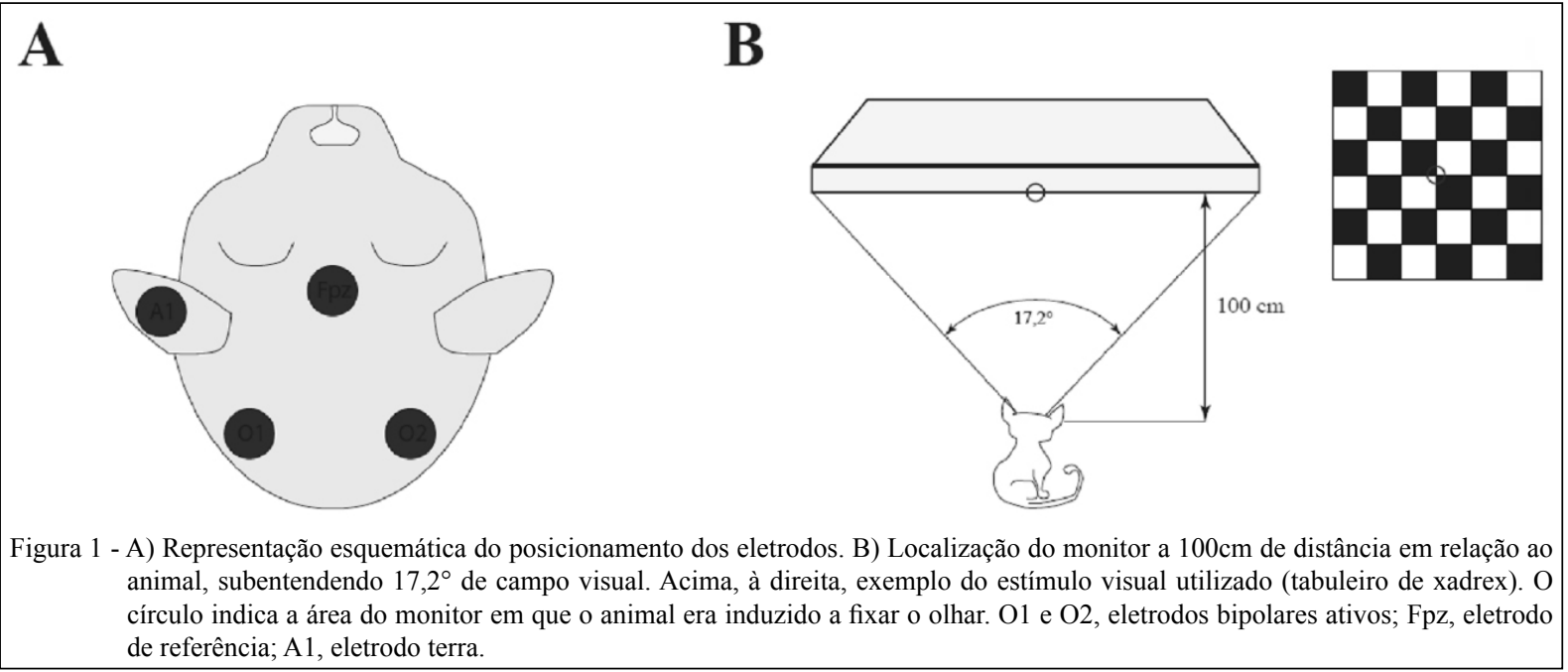

considerados os valores de amplitude $(\mu \mathrm{V})$ e tempo implícito (ms) dos picos N75 e P100 para cada frequência espacial. Os resultados foram expressos como o cálculo das médias e desvios padrão.

\section{Análise estatística}

Os valores de amplitude e tempo implícito dos picos N75 e P100 foram analisados com o programa BioEstat 5.3. A normalidade dos dados foi verificada por meio do teste de ShapiroWilk. As diferenças foram submetidas ao teste ANOVA e os valores de $\mathrm{P}<0,05$ foram considerados significantes. Quando os valores não seguiam uma distribuição normal, o teste de Kruskal-Wallis foi utilizado. Uma correlação de Pearson foi usada para verificar se as mudanças encontradas entre os tempos implícitos e amplitudes dos picos estavam relacionadas. Todos os resultados são expressos como média \pm erro padrão da média (SEM).

\section{RESULTADOS}

Um característico PVE-PR, formado por três picos, denominados negativos e positivos, N75, P100 e N135 foram identificados em todos os ângulos em que os estímulos foram apresentados. A parte mais proeminente e constante do PVE-PR foi o complexo N75 e P100, apenas os tempos implícitos e amplitudes destes picos foram analisados (Figura 2, Tabela 1).

Com a mudança de tamanho do ângulo de estímulo apresentado, os picos de tempo implícito e amplitude do P100 apresentaram uma discreta variação. Aos 32'de arco, a amplitude de P100 atingiu seu pico máximo, com $14,94 \pm 1,13 \mu \mathrm{V}$ e volta a diminuir quando subtendido aos 16', enquanto que o tempo implícito máximo de P100
$(116,73 \pm 3,33 \mathrm{~ms})$ foi observado aos 64' de arco. Não foi observada correlação entre os valores médios dos tempos implícitos e amplitudes de P100 (r=0,067). A deflexão do registro N75 não mostrou nenhuma variação significativa de sua amplitude. No entanto, aos 16' de arco, o tempo implícito da onda N75 mostrou-se reduzido, em comparação às demais frequências espaciais, sendo significativamente menor que 32 e 64' de arco. Não houve correlação estatística entre estes dados $(r=0,26)$ e nem diferença significativa entre todos os valores.

Os ângulos visuais que desencadearam um maior pico de amplitude e tempo implícito para P100 foi o de 32' e 64' de arco, respectivamente. Nessas frequências espaciais, oregistrodoPVE-PRapresentou um traçado homogêneo e com picos bem definidos.

Em gatos adultos, um PVE-PR pode ser obtido com estímulos subtendidos entre ângulos grandes como 64' e pequenos como 16'.

\section{DISCUSSÃO}

Os procedimentos mais comumente utilizados para análise da visão durante a rotina clínica oftálmica são aqueles que testam a capacidade da retina para detectar movimentos, como resposta de ameaça e capacidade para seguir um algodão (OFFRI, 1999) ou testes comportamentais caracterizados pela tomada de decisão pelo animal, baseada em indícios visuais (GEARHART et al., 2008). Nossos resultados contribuem para a normatização de um exame objetivo, o PVE-PR com tabuleiro de xadrez em gatos adultos sem o uso de midriáticos e sedativos. Deve-se ressaltar que a execução do PVE-PR sem a utilisação de midriáticos ou sedativos contribui para a obtenção de dados mais precisos que descrevam 


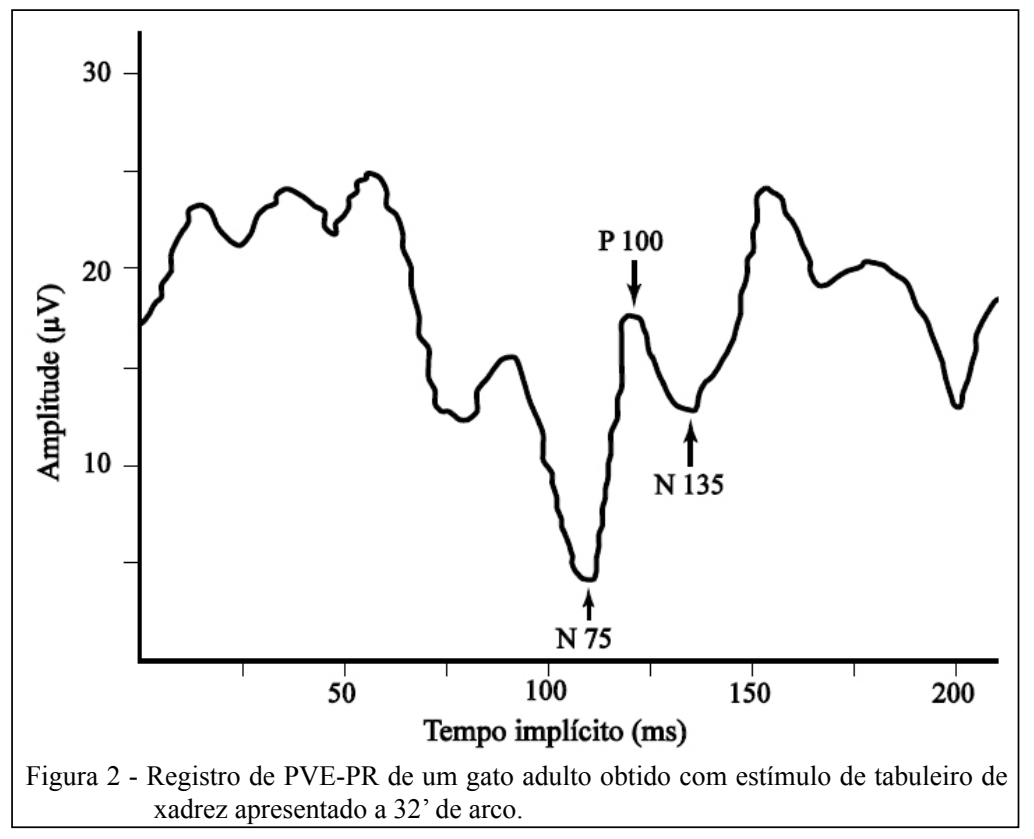

a função visual do animal. No entanto, devemos ressaltar que a execução do exame sem o uso de drogas requer alguns cuidados, pois movimentos das orelhas e enrugamento da pele fazem com que os eletrodos se desloquem de sua posição ideal. Além disso, animais não colaborativos, principalmente gatos, que desviam frequentemente a atenção do estímulo visual, constituem um obstáculo importante para a realização do exame. Nesses casos, o animal precisa ser tranquilizado e sua atenção ser direcionada ao centro do monitor de vídeo com objetos pequenos, como uma pequena argola presa a um fio de algodão. Esta particularidade já havia sido relatada em um estudo semelhante feito em cães (MORENOCARMONA, 2006). Por último, destaca-se ainda a necessidade da presença de um membro da equipe, atento aos olhos do paciente que, algumas vezes, pode fechar as pálpebras.

O PVE reflete primariamente a atividade elétrica gerada pela estimulação do campo visual central que está localizada mais próxima à superfície no pólo occipital, visto que a representação do campo periférico está mais profundamente localizada dentro do sulco calcarino. Estímulos de tabuleiro de xadrez pequenos podem ser resolvidos somente pelo campo visual central (BRIGELL, 2001).

Em nossos resultados, o menor tamanho dos elementos do padrão de estímulo 16 Minutos de Arco deu origem a PVE-PR de menor tempo implícito para a onda N75. A redução do tempo implícito, observado no pico N75 a 16' de arco, poderia estar relacionada à ativação da via magnocelular do corpo geniculado lateral, caracterizada por possuir uma velocidade de condução neural mais rápida que a via parvocelular (LEE et al., 1989). No entanto, a associação entre o tempo implícito reduzido da onda N75, encontrado no presente estudo, e a via magnocelular resta a ser comprovada, variando-se outros parâmetros do estímulo visual, como o contraste e frequência temporal.

Sabe-se que estímulos de alta frequência ativam as duas vias (LEE et al., 1990; SHAPLEY, 1990). Esta variabilidade reafirma a importância de se

Tabela 1 - Valores médios e seus respectivos erros padrões (SEM) das amplitudes e tempos implícitos dos picos N75 e P100 do PVE-PR de tabuleiro de xadrez em gatos adultos, com resposta a variados ângulos visuais.

\begin{tabular}{|c|c|c|c|c|c|c|c|c|c|c|c|}
\hline \multicolumn{2}{|c|}{ Ângulo visual } & \multicolumn{2}{|c|}{ - } & \multicolumn{2}{|c|}{ 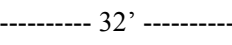 } & \multicolumn{2}{|c|}{ 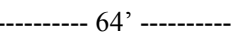 } & \multicolumn{2}{|c|}{-------130' ------- } & \multicolumn{2}{|c|}{ 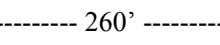 } \\
\hline \multirow{3}{*}{$\begin{array}{l}\text { Tempo } \\
\text { implícito }\end{array}$} & & N75 & P100 & N75 & P100 & N75 & P100 & N75 & P100 & N75 & P100 \\
\hline & Média & 74,98 & 112,9 & 91,87 & 115,4 & 92,13 & 116,7 & 86,05 & 110,2 & 78,15 & 107,1 \\
\hline & SEM & 4,30 & 3,35 & 4,68 & 4,82 & 3,42 & 3,33 & 4,64 & 3,04 & 2,57 & 3,40 \\
\hline \multirow{2}{*}{ Amplitude } & Média & 4,6 & 12,49 & 5,12 & 14,94 & 4,57 & 12,34 & 4,14 & 14,54 & 4,32 & 12,26 \\
\hline & SEM & 0,47 & 0,86 & 0,64 & 1,13 & 0,39 & 0,98 & 0,68 & 1,63 & 0,72 & 0,74 \\
\hline
\end{tabular}


realizar estudo normativo próprio para o PVE-PR em cada serviço de Eletrodiagnóstico (ODOM, 2010).

O Potencial Visual Evocado (PVE) permite avaliar a função e integridade das estruturas corticais e subcorticais da via visual (FELGUEIRA et al., 2005). A principal indicação dos PVE em seres humanos consiste do diagnóstico de enfermidades que produzam lesões desmielinizantes no nervo óptico, entre essas a esclerose múltipla, lesões compressivas (tumores) ou não compressivas do nervo óptico (neurite óptica isquêmica, ambliopias nutricionais e tóxicas), alcoolismo crônico, doenças afetando difusamente o sistema nervoso, como encefalopatias de origem metabólica, anóxica e ainda a neuropatia diabética (CHIAPPA, 1997). Gatos que foram inoculados com o vírus da imunodeficiência felina apresentaram registros de PVE com tempos implícitos aumentados (PHIPPS et al., 2000).

As medidas de acuidade visual obtidas por métodos eletrofisiológicos também estão sujeitas a subestimar a real capacidade do paciente em executar a tarefa visual que lhe é apresentada. Isto se deve à presença inevitável de ruído em todos os testes, sejam eles comportamentais ou eletrofisiológicos, que vão desde a atividade muscular, cooperação, acomodação imprecisa e movimento dos olhos (HAMER \& MAYER, 1994). Associado a estes fatores, o equipamento utilizado no presente estudo estava limitado a estímulos de 16 minutos de arco, quando apresentados a $100 \mathrm{~cm}$ de distância do monitor, obtendo-se ainda consideráveis valores de amplitude e tempo implícito. Devido ao limitado espectro de frequências espaciais fornecidas pelo equipamento utilizado $(0.11$ à $1.87 \mathrm{cpd})$, não foi possível estabelecer valores de AV para os animais testados. Outros pesquisadores demonstraram que a AV de gatos encontra-se entre 3,75-7,5 ciclos por grau, portanto bastante acima dos valores de frequência espacial utilizados no presente estudo (BERKLEY \& WATKINS, 1973; OFRI, 1999).

\section{CONCLUSÃO}

O exame de PVE-PR com tabuleiro de xadrez em gatos domésticos adultos mostrouse simples, não invasivo e não requer o uso de sedativos e midriáticos. Respostas visuais evocadas características puderam ser obtidas com a apresentação de diferentes frequências espaciais, contendo estímulos de baixa (64' de arco) e alta frequência (16' de arco). O presente estudo fornece dados importantes para a normatização do PVEPR em gatos adultos e serve como referência para a realização de estudos em gatos portadores de alguma oftalmopatia ou doença sistêmica, com repercussão neuroftalmológica.

\section{COMITÊ DE ÉTICA E BIOSSEGURANÇA}

Este trabalho teve aprovação da Comissão de Ética no Uso de Animais da Universidade Federal Rural de Pernambuco (UFRPE), sob registro ํㅜ 23082.001546/2014-24.

\section{AGRADECIMENTOS}

À Coordenação de Aperfeiçoamento de Pessoal de Nível Superior (CAPES) pelo apoio financeiro.

\section{REFERÊNCIAS}

BERKLEY, M.A.; WATKINS, D.W. Grating resolution and refraction in the cat estimated from evoked cerebral potentials. Vision research, v.13, n.2, p.403-415, 1973. Disponível em: <http://www.sciencedirect.com/science/article/ pii/004269897390117X\#>. Acesso em: 10 jan. 2014.

BRIGELL, M.G. The visual evoked potential. In: FISHMAN, G.A. et al. (Ed.). Electrophysiologic testing in disorders of the retina, optic nerve and visual pathway. The Foundation of the American Academy of Ophthalmology, 2001. p.237-279.

CHIAPPA, K.H.; HILL, R.A. Pattern-shift visual evoked potentials: interpretation. In: CHIAPPA, K.H. (Ed.). Evoked potentials in clinical medicine. Lippincott-Raven Publishers, 1997. Cap.3, p.95-146.

FELGUEIRA, R.M.; GUILHOTO, L.M. de F.F. Estudo normativo do potencial evocado visual por padrão reverso. Journal of Epilepsy and Clinical Neurophysiology, v.11, n.4, p.171-175, 2005. Disponível em: <http://www.scielo.br/pdf/jecn/v11n4/ a04v11n4.pdf>. Acesso em: 13 jan. 2014.

GEARHART, P.M. et al. A novel method for objective vision testing in canine models of inherited retinal disease. Investigative Ophthalmology \& Visual Science, v.49, n.8, p.3568-3576, 2008.

HAMER, R.D.; MAYER, D.L. The development of spatial vision. In: ALBERT, D.M.; JAKOBIEC, F.A. (Ed.). Principles and practice of ophthalmology: basic sciences. Saunders, 1994. p.578-608.

ITOH, Y. et al. Pattern-stimulated visual evoked potential in dog: changes in elicited response with pattern size and calculation of visual acuity. Journal of Veterinary Medical Science/the Japanese Society of Veterinary Science, v.72, n.11, p.14491453, 2010. Disponível em: <http://www.ncbi.nlm.nih.gov/ pubmed/20644341>. Acesso em: 13 jan. 2014. doi: 10.1292/ jvms.10-007.

JACQUES, C. et al. Long-term effects of prenatal omega-3 fatty acid intake on visual function in school-age children. Journal of Pediatrics, v.158, n.1, p.83-90.e1, 2011.

KORNMEIER, J. et al. A different view on the checkerboard? Alterations in early and late visually evoked eeg potentials in asperger observers. PLoS ONE, v.9, n.3, p.e90993, 2014. 
LEE, BB. et al. Sensitivity of macaque retinal ganglion cells to chromatic and luminance flicker. Journal of Physiology, v.414, p.223243, 1989. Disponível em: <http://www.ncbi.nlm.nih.gov/pmc/articles/ PMC1189139/pdf/jphysiol00485-0228.pdf>. Acesso em: 13 jan. 2014.

LEE, B. B. et al. Luminance and chromatic modulation sensitivity of macaque ganglion cells and human observers. Journal of the Optical Society of America, n.7, p.2223-2236, 1990. Disponível em: <http:/ macboy.uchicago.edu/ eye1/PDF\%20files/Lee.et.al.\%2090.pdf>. Acesso em: 13 jan. 2014.

MILLER, P.E.; MURPHY, C.J. Vision in dogs. Journal of the American Veterinary Medical Association, v.207, p.1623-1634, 1995. Disponível em: <http://www.smartpawsdogagility.com/ userFiles/dogvisionmillermurphy.pdf>. Acesso em: 13 jan. 2014.

MORENO-CARMONA, F.M. et al. Grating visual acuity by sweep visually evoked potentials: methodology standardization for use in dogs. Brazilian Journal of Veterinary Research and Animal Science, v.43, supl, p. 86-92, 2006. Disponível em: <http://www. bv.fapesp.br/pt/publicacao/51186/grating-visual-acuity-sweepvisually/>. Acesso em: 13 jan. 2014.

MURPHY, C.J. et al. Effect of optical defocus on visual acuity in dogs. American Journal of Veterinary Research, v.58, n.4, p.414-418, 1997. Disponível em: <http://www.ncbi.nlm.nih.gov/ pubmed/9099390>. Acesso em: 13 jan. 2014.

ODOM, J.V. et al. ISCEV standard for clinical visual evoked potentials (2009 update). Documenta ophthalmologica, v.120, n.1, p.111-119, 2010. Disponível em: <http://www.ncbi.nlm.nih. gov/pubmed/19826847>. Acesso em: 13 jan. 2014. doi: 10.1007/ s10633-009-9195-4.

OFRI, R. Optics and physiology of vision. In: GELATT, K.N Veterinary ophthalmology. New Jersey: Wiley-blackwell, 1999. Cap.4, p.112-217.

OYAMADA, M.K. Eletrofisiologia em neuroftalmologia. In: RODRIGUES-ALVES, C.A. Neuroftalmologia. São Paulo: Roca, 2000. p.343-362.
PHIPPS, A.J. et al. Neurophysiologic and immunologic abnormalities associated with feline immunodeficiency virus molecular clone FIVPPR DNA inoculation. Journal of acquired immune deficiency syndromes, v.23, n.1, p.8-16, 2000. Disponível em: <http://www. ncbi.nlm.nih.gov/pubmed/10708051>. Acesso em: 13 jan. 2014.

REN, J.C et al. Retinal degeneration in the nervous mutant mouse. III. Electrophysiological studies of the visual pathway. Experimental eye research, v.70, n.4, p.467-473, 2000. Disponível em: <http://www.ncbi.nlm.nih.gov/pubmed/10865995>. Acesso em: 13 jan. 2014. Doi: 10.1006 exer.1999.0804.

ROSOLEN, S.G. et al. The ERG of the Beagle dog: evidence associating a post b-wave negativity with the Tapetum Lucidum. Documenta ophthalmologica, v.110, n.2-3, p.145153, 2005. Disponível em: <http://www.ncbi.nlm.nih.gov/ pubmed/16328922>. Acesso em: 13 jan. 2014. doi: DOI 10.1007/ s10633-005-1816-y.

SALOMÃO, S.R. Desenvolvimento da acuidade visual de grades. Psicologia USP, v.18, n.2, p.63-81, 2007. Disponível em: <http:// www.revistas.usp.br/psicousp/article/view/41921/45589>. Acesso em: 13 jan. 2014.

SCHMIDT, K.E. et al. Processing deficits in primary visual cortex of amblyopic cats. Journal of Neurophisiology, v.91, p.1661-1671, 2004. Disponível em: <http://jn.physiology.org/ content/91/4/1661.long>. Acesso em: 13 jan. 2014. doi: 10.1152/ jn.00878.2003.

SHAPLEY, R. Visual sensitivity and parallel retinocortical channels. Annual Review of Psychology, v.41, p.635-658, 1990. Disponível em: <http://www.annualreviews.org/doi/abs/10.1146/ annurev.ps.41.020190.003223>. Acesso em: 13 jan. 2014. doi: 10.1146/annurev.ps.41.020190.003223.

TANAKA, T. et al. Studies on the visual acuity of dogs using shape discrimination learning. Animal Science Journal, v.71, n.6, p.614620, 2000. Disponível em: <https:/www.jstage.jst.go.jp/article/ chikusan1924/71/6/71_6_614/_pdf>. Acesso em: 13 jan. 2014. 